\title{
ON LACUNARY INVARIANT SEQUENCE SPACES DEFINED BY A SEQUENCE OF MODULUS FUNCTIONS
}

\author{
VATAN KARAKAYA AND NECIP SIMSEK
}

\begin{abstract}
The purpose of this paper is to introduce and study some sequence spaces which are defined by combining the concepts of lacunary convergence, invariant mean and the sequence of modulus functions We also examine some topological properties of these spaces.
\end{abstract}

\section{Introduction}

Let $\ell_{\infty}$ and $c$ denote the Banach spaces of real bounded and convergent sequences $x=\left(x_{k}\right)$ normed by $\|x\|=\sup \left|x_{k}\right|$, respectively.

Let $\sigma$ be a mapping of the set of positive integers into itself. A continuous linear functional $\phi$ on $\ell_{\infty}$, the space of real bounded sequences, is said to be an invariant mean or $\sigma$-mean if and only if

i. $\phi(x) \geq 0$ when the sequence $x=\left(x_{n}\right)$ has $x_{n} \geq 0$ for all $n$,

ii. $\phi(e) \geq 0$, where $e=(1,1,1, .$.$) and,$

iii. $\phi\left(x_{\sigma(n)}\right)=\phi(x)$ for all $x \in \ell_{\infty}$.

Let $V_{\sigma}$ denote the set of bounded sequence all of whose invariant means are equal. In particular, if $\sigma$ is the translation $n \rightarrow n+1$, then a $\sigma$-mean reduce to a Banach limit (see, Banach [1]) and set $V_{\sigma}$ reduce to $\hat{c}$, the spaces of all almost convergent sequences (see, Lorentz [7] ).

If $x=\left(x_{n}\right)$, write $T x=T x_{n}=\left(x_{\sigma(n)}\right)$. It can be shown (Schaefer [16]) that $V_{\sigma}=\left\{x \in \ell_{\infty}: \lim _{k} t_{k n}(x)=\ell\right.$, uniformly in $\left.\mathrm{n},\right\} \ell=\sigma-\lim x$, where

$$
t_{k n}(x)=\frac{x_{n}+x_{\sigma^{1}(n)}+\ldots+x_{\sigma^{k}(n)}}{k+1} .
$$

Here $\sigma^{k}(n)$ denote the $k^{\text {th }}$ iterate of the mapping $\sigma$ at $n$. The mapping $\sigma$ is one to one and such that $\sigma^{k}(n) \neq n$ for all positive integers $n$ and $k$. Thus a $\sigma$ - mean $\phi$ extends the limit functional on $c$, the spaces of convergent sequence, in the sense that $\phi(x)=\lim x$ for all $x \in c$. (see, Mursaleen [11]).

We call $V_{\sigma}$ as the space of $\sigma$-convergent sequences.

A sequence $x=\left(x_{k}\right)$ is said to be strongly $\sigma$-convergent (Mursallen [12]) if there exists a number $\ell$ such that $\lim _{k} \frac{1}{k} \sum_{j=1}^{k}\left|x_{\sigma^{j}(n)}-\ell\right|=0$ uniformly in $n$.

Received by the editors: 04.03.2002.

2000 Mathematics Subject Classification. Primary 43A10, 43A20; Secondary 46H20.

Key words and phrases. Modulus functions. 
We denote $\left[V_{\sigma}\right]$ as the set of all strongly $\sigma$-convergent sequences. In case $\sigma(n)=n+1,\left[V_{\sigma}\right]$ reduce to $[\hat{c}]$, the space of all strong almost convergent sequence (Maddox [8]).

Also the strongly almost convergent sequences was studied by Freedman et all [4], independently.

By a lacunary $\theta=\left(k_{r}\right) ; r=0,1,2, \ldots$ where $k_{0}=0$, we shall mean an increasing sequence of non-negative integers with $k_{r}-k_{r-1} \rightarrow \infty$ as $r \rightarrow \infty$. The intervals determined by $\theta$ will be denoted by $I_{r}=\left(k_{r-1}, \mathrm{k}_{\mathrm{r}}\right]$ and $h_{r}=k_{r}-k_{r-1}$. The ratio $\frac{k_{r}}{k_{r-1}}$ will be denoted by $q_{r}$. The space of lacunary strongly convergent sequence $N_{\theta}$ was defined by Freedman et al [4] as:

$$
N_{\theta}=\left\{x=\left(x_{k}\right): \lim _{r} \frac{1}{h_{r}} \sum_{k \in I_{r}}\left|x_{k}-\ell\right|=0, \text { for some } \ell\right\}
$$

Recently, the concept of lacunary strong $\sigma$-convergence was introduced by Savas [14] which is a generalization of the idea of lacunary strong almost convergence due to Das and Mishra [2].

A modulus function $f$ is a function from $[0, \infty)$ to $[0, \infty)$ such that

i. $f(x)=0$ if and only if $x=0$

ii. $f(x+y) \leq f(x)+f(y)$, for all $x, y>0$

iii. $f$ is increasing,

iv. $f$ is continuous from the right at zero.

Since $|f(x)-f(y)| \leq f(|x-y|)$, it follows from conditions (ii) and (iv) that $f$ is continuous everywhere on $[0, \infty)$.

A modulus function may be bounded or unbounded. For example, $f(t)=\frac{t}{t+1}$ is bounded but $f(t)=t^{p}(0<p \leq 1)$ is unbounded.

Ruckle [13] and Maddox [9], Savas [15] and other authors used modulus function to construct new sequence spaces.

Recently, Kolk ( $[6],[7])$ gave an extension of $X(f)$ by considering a sequence of moduli $F=\left(f_{k}\right)$ i.e.,

$$
X\left(f_{k}\right)=\left\{x=\left(x_{k}\right):\left(f_{k}\left(\left|x_{k}\right|\right)\right) \in X\right\}
$$

In this paper by combining lacunary sequence, invariant mean and a sequence of modulus functions, we define the following new sequence spaces:

$$
\begin{gathered}
{\left[w_{\sigma}^{0}, F\right]_{\theta}=\left\{x: \lim _{r} \frac{1}{h_{r}} \sum_{k \in I_{r}} f_{k}\left(\left|t_{k n}(x)\right|\right)=0, \text { uniformly in } \mathrm{n}\right\}} \\
{\left[w_{\sigma}, F\right]_{\theta}=\left\{x: \lim _{r} \frac{1}{h_{r}} \sum_{k \in I_{r}} f_{k}\left(\left|t_{k n}(x-l)\right|\right)=0, \text { uniformly in } \mathrm{n} \text {, for some l }\right\}} \\
{\left[w_{\sigma}^{\infty}, F\right]_{\theta}=\left\{x: \sup _{r, n} \frac{1}{h_{r}} \sum k \in I_{r} f_{k}\left(\left|t_{k n}(x)\right|\right)<\infty\right\}} \\
{\left[w_{\sigma}, F\right]=\left\{x: \lim _{r} \frac{1}{m} \sum_{k=1}^{m} f_{k}\left(\left|t_{k n}(x-l)\right|\right)=0, \text { uniformly in } \mathrm{n}, \text { for some } 1\right\}}
\end{gathered}
$$

Some sequence spaces are obtained by specializing $F, \theta, \sigma$. For example, if 
$\theta=\left(2^{r}\right), \quad \sigma(n)=n+1$ and $f_{k}(x)=x$ for all $k$, then $\left[w_{\sigma}, F\right]_{\theta}=\hat{w}$ (see, Das and Sahoo [3]). If $\sigma(n)=n+1$ and $f_{k}(x)=f$ for all $k$, then $\left[w_{\sigma}, F\right]_{\theta}=[\hat{w}(f)]_{\theta}$ and $\left[w_{\sigma}, F\right]=[\hat{w}(f)]$ (see, Mursaleen and Chishti [12]).

When $\sigma(n)=n+1$, the spaces $\left[w_{\sigma}^{0}, F\right]_{\theta},\left[w_{\sigma}, F\right]_{\theta}$ and $\left[w_{\sigma}^{\infty}, F\right]_{\theta}$ reduce to the spaces $\left[\hat{w}_{0}, F\right]_{\theta},[\hat{w}, F]_{\theta}$ and $\left[\hat{w}_{\infty}, F\right]_{\theta}$ respectively, where

$$
[\hat{w}, F]_{\theta}=\left\{x=\left(x_{k}\right): \lim _{r} \frac{1}{h_{r}} \sum_{k \in I_{r}} f_{k}\left(\left|d_{k n}(x-l)\right|\right)=0,\right.
$$

uniformly in n, for somel\}

and

$$
d_{n k}(x)=\frac{x_{n}+x_{n+1}+\ldots+x_{n+k}}{k+1}
$$
$\left[w_{\sigma}^{\infty}, F\right]$.

If $\theta=\left(2^{r}\right)$, then $\left[w_{\sigma}^{0}, F\right]_{\theta}=\left[w_{\sigma}^{0}, F\right],\left[w_{\sigma}, F\right]_{\theta}=\left[w_{\sigma}, F\right]$ and $\left[w_{\sigma}^{\infty}, F\right]_{\theta}=$

\section{Main Results}

We have

Theorem 2.1. For any a sequence of modulus functions $F=\left(f_{k}\right),\left[w_{\sigma}^{0}, F\right]_{\theta},\left[w_{\sigma}, F\right]_{\theta}$, $\left[w_{\sigma}^{\infty}, F\right]_{\theta}$ and $\left[w_{\sigma}, F\right]$ are linear spaces over the set of complex numbers.

Proof. We shall prove the result only for $\left[w_{\sigma}^{0}, F\right]_{\theta}$. The others can be treated similarly. Let $x, y \in\left[w_{\sigma}^{0}, F\right]_{\theta}$ and $\alpha, \beta \in \mathrm{C}$. Then there exist integers $H_{\alpha}$ and $K_{\beta}$ such that $|\alpha|<H_{\alpha}$ and $|\beta|<K_{\beta}$. We have

$$
\begin{gathered}
\frac{1}{h_{r}} \sum_{k \in I_{r}} f_{k}\left(\left|t_{k n}(\alpha x-\beta y)\right|\right) \leq H_{\alpha} \frac{1}{h_{r}} \sum_{k \in I_{r}} f_{k}\left(\left|t_{k n}(x)\right|\right) \\
+K_{\beta} \frac{1}{h_{r}} \sum_{k \in I_{r}} f_{k}\left(\left|t_{k n}(y)\right|\right)
\end{gathered}
$$

This implies $\alpha x+\beta y \in\left[w_{\sigma}^{0}, F\right]_{\theta}$

We will now give a lemma.

Lemma 2.2. Let $f$ be a modulus and let $0<\delta<1$. Then for each $\left|t_{k n}(x)\right|>\delta$ for all $k$ and $n$ we have

$$
f\left(\left|t_{k n}(x)\right|\right) \leq 2 f(1) \delta^{-1}\left|t_{k n}(x)\right|
$$

Proof.

$$
\begin{gathered}
f\left(\left|t_{k n}(x)\right|\right) \leq f\left(1+\left[\frac{\left|t_{k n}(x)\right|}{\delta}\right]\right) \leq f(1)+f\left(\left[\frac{\left|t_{k n}(x)\right|}{\delta}\right]\right) \\
\leq f(1)\left(1+\frac{\left|t_{k n}(x)\right|}{\delta}\right) \leq 2 f(1) \delta^{-1}\left|t_{k n}(x)\right|
\end{gathered}
$$

Theorem 2.3. For a sequence of modulus functions $F=\left(f_{k}\right)$ and any lacunary sequence $\theta=\left(k_{r}\right)$,

$$
\left[w_{\sigma}, F\right]_{\theta} \subset\left[w_{\sigma}^{\infty}, F\right]_{\theta} .
$$


Proof. Let $F=\left(f_{k}\right)$ be a sequence of modulus functions and $x \in\left[w_{\sigma}, F\right]_{\theta}$. Put $\sup _{k} f_{k}(1)=M$. We can write

$$
\begin{gathered}
\frac{1}{h_{r}} \sum_{k \in I_{r}} f_{k}\left(\left|t_{k n}(x)\right|\right) \leq \frac{1}{h_{r}} \sum_{k \in I_{r}} f_{k}\left(\left|t_{k n}(x-l)\right|\right)+\frac{1}{h_{r}} \sum_{k \in I_{r}} f_{k}(|l|) \\
\leq \frac{1}{h_{r}} \sum_{k \in I_{r}} f_{k}\left(\left|t_{k n}(x-l)\right|\right)+T_{l} M
\end{gathered}
$$

where $T_{l}$ is integer number such that $|l|<T_{l}$. Hence $x \in\left[w_{\sigma}^{\infty}, F\right]_{\theta}$. and $\left[w_{\sigma}, F\right]$.

Now for any lacunary sequence $\theta=\left(k_{r}\right)$, we give connection between $\left[w_{\sigma}, F\right]_{\theta}$

Theorem 2.4. Let $\theta=\left(k_{r}\right)$ be a lacunary sequence with $\liminf _{r} q_{r}>1$. Then for sequence of modulus functions $F=\left(f_{k}\right)$,

$$
\left[w_{\sigma}, F\right] \subset\left[w_{\sigma}^{\prime} F\right]_{\theta}
$$

Proof. Suppose that $\liminf q_{r}>1$, then there exists $\delta>0$ such that $q_{r}>1+\delta$ for all $r$. Then for $x \in\left[w_{\sigma}, \stackrel{r}{F}\right]$, we write

$$
\begin{aligned}
\frac{1}{k_{r}} \sum_{k=1}^{k_{r}} f_{k}\left(\left|t_{k n}(x-l)\right|\right) & \geq \frac{1}{k_{r}} \sum_{k=1}^{k_{r}} f_{k}\left(\left|t_{k n}(x-l)\right|\right)+\frac{1}{k_{r}} \sum_{k=1}^{k_{r-1}} f_{k}\left(\left|t_{k n}(x-l)\right|\right) \\
& =\frac{1}{k_{r}} \sum_{k \in I_{r}} f_{k}\left(\left|t_{k n}(x-l)\right|\right) \\
\geq & \frac{\delta}{1+\delta} \frac{1}{h_{r}} \sum_{k \in I_{r}} f_{k}\left(\left|t_{k n}(x-l)\right|\right)
\end{aligned}
$$

By taking limit as $r \rightarrow \infty$ uniformly in, hence we obtain $x \in\left[w_{\sigma}, F\right]_{\theta}$. This completes the proof.

Theorem 2.5. Let $\theta=\left(k_{r}\right)$ be a lacunary sequence with $\lim \sup q_{r}<\infty$. Then for any sequence of modulus functions $F=\left(f_{k}\right)$,

$$
\left[w_{\sigma}, F\right]_{\theta} \subset\left[w_{\sigma}, F\right]
$$

Proof. If $\lim \sup q_{r}<\infty$, there exists $H>0$ such that $q_{r}<H$ for all $r \geq 1$. Let $x \in\left[w_{\sigma}, F\right]_{\theta}$ and $\varepsilon>0$. There exists $R>0$ such that for every $j \geq R$ and all $n$

$$
A_{j}=\frac{1}{h_{j}} \sum_{k \in I_{j}} f_{k}\left(\left|t_{k n}(x-l)\right|\right)<\varepsilon .
$$

We can also find $M>0$ such that $A_{j}<K$ for all $j=1,2, \ldots$. Now let $\mathrm{m}$ be any integer with $k_{r-1}<m \leq k_{r}$, where $r>R$. We have

$$
\begin{gathered}
\frac{1}{m} \sum_{k=1}^{m} f_{k}\left(\left|t_{k n}(x-l)\right|\right) \leq \frac{1}{k_{r-1}} \sum_{k=1}^{k_{r}} f_{k}\left(\left|t_{k n}(x-l)\right|\right) \\
=\frac{1}{k_{r-1}} \sum_{j=1}^{r} \sum_{k \in I_{j}} f_{k}\left(\left|t_{k n}(x-l)\right|\right)
\end{gathered}
$$




$$
\begin{gathered}
=\frac{1}{k_{r-1}} \sum_{j=1}^{R} \sum_{k \in I_{j}} f_{k}\left(\left|t_{k n}(x-l)\right|\right)+\frac{1}{k_{r-1}} \sum_{j=R+1}^{r} \sum_{k \in I_{j}} f_{k}\left(\left|t_{k n}(x-l)\right|\right) \\
\leq \frac{1}{k_{r-1}}\left(\sup _{j \leq R} A_{j}\right) k_{R}+\frac{1}{k_{r-1}} \varepsilon\left(\sum_{j=R+1}^{r} h_{j}\right) \\
\leq \frac{1}{k_{r-1}} M k_{R}+\frac{1}{k_{r-1}} \varepsilon\left(h_{R+1}+h_{R+2}+\ldots+h_{r}\right) \\
\leq \frac{1}{k_{r-1}} M k_{R}+\varepsilon H
\end{gathered}
$$

Since $k_{r-1} \rightarrow \infty$ as $r \rightarrow \infty$, it follows that

$$
\frac{1}{m} \sum_{k=1}^{m} f_{k}\left(\left|t_{k n}(x-l)\right|\right) \rightarrow 0
$$

uniformly in $n$ and consequently $x \in\left[w_{\sigma}, F\right]$. Hence the proof completes.

Theorem 2.6. Let $\theta=\left(k_{r}\right)$ be a lacunary sequence $1<\liminf _{r} q_{r} \leq \limsup q_{r}<\infty$. Then for any sequence of modulus functions $F=\left(f_{k}\right)$,

$$
\left[w_{\sigma}, F\right]_{\theta}=\left[w_{\sigma}, F\right]
$$

Proof. Theorem 2.6 follows the theorems 2.5 and 2.4 .

\section{References}

[1] S. Banach, Theorie des operations linearies, Warszawa, 1932.

[2] G. Das, S. Mishra, Lacunary distribution of sequences, Indian J. Pure Apll. Math., 20(1)(1989), 64-74.

[3] G. Das, A. K. Sahoo, On some sequence spaces, J. Math. Anal. Appl., 164(1992), 381-398.

[4] A. R. Freedman, J. J. Sember, M. Raphael, Some Cesaro-type summability, Proc. London Math. Soc., 37(3)(1978), 508-520.

[5] E. Kolk, On strong boundedness and summability with respect to a sequence of moduli, Acta et. Comment. Univ. Tartu, 960(1993), 41-50.

[6] E. Kolk, Inclusion theorems for some sequence spaces defined by a sequence of moduli, Acta et. Comment. Univ. Tartu, 970(1994), 65-72.

[7] G. G. Lorentz, A contribution to the theory of divergent series, Acts Math. 80(1948), 167-190.

[8] I. J. Maddox, On strong almost convergence, Math. Proc. Camb. Phil. Soc. 85(1979), 345-350.

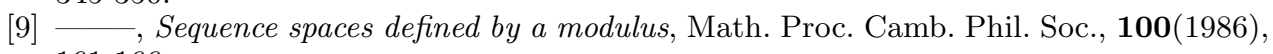
161-166.

[10] Mursaleen, On some new invariant matrix methods of summability, Quart. J. Math. Oxford, 34(1983), 77-86.

[11] -, Matrix transformation between some new sequence spaces, Houston J. Math., 9(1983), 505-509.

[12] Mursaleen, T. A. Chishti, Some spaces of lacunary sequences defined by the modulus, J. Analysis, 4(1996), 153-159.

[13] W. H. Ruckle, FK spaces in which the sequence of coordinate vectors is bounded, Can. J. Math. 25(1973), 973-978.

[14] E. Savas, On lacunary strong $\sigma$-convergence, Indian J. Pure Appl. Math., 21(4) (1990), 359-365.

[15] E. Savas, On some generalized sequence spaces defined by a modulus, Indian J. Pure and Apl. Math., 38(1)(1999), 459-464. 
VATAN KARAKAYA AND NECIP SIMSEK

[16] P. Schaefer, Infinite matrices and invariant means, Proc. Amer. Math. Soc., 36(1972), 104-110.

Yüzüncü Yil University, FacUlty of ARts and Sciences,

Department of MATHEMATICS, 65080 VAN, TURKEY

E-mail address: vkkaya@yahoo.com, nsimsek@yyu.edu.tr 